\title{
Original Article (short paper) \\ Impacts of low or vigorous levels of physical activity on body composition, hemodynamics and autonomic modulation in Down syndrome subjects
}

\author{
Antonio Silva Cunha ${ }^{1}$, Antonio Carlos Silva-Filho ${ }^{1}$, Carlos José Dias ${ }^{1,2}$, Rafael Durans ${ }^{1}$, Janaína de Oliveira Brito- \\ Monzani $^{1}$, Bruno Rodrigues ${ }^{3}$, Cristiano Teixeira Mostarda ${ }^{1}$ \\ ${ }^{1}$ Universidade Federal do Maranhão, UFMA, Laboratory of Cardiovascular Adaptations to Exercise, LACO-

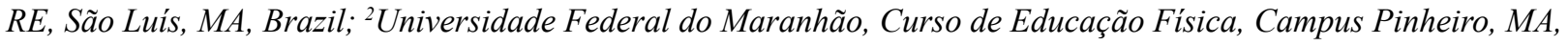 \\ Brazil, ${ }^{3}$ Universidade Estadual de Campinas, UNICAMP, Faculty of Physical Education, Campinas, SP, Brazil
}

\begin{abstract}
Aim: We aimed to evaluate the effect of low and vigorous levels of physical activity in body composition, hemodynamics and autonomic modulation in subjects with Down syndrome (DS). Methods: We evaluated 13 healthy subjects without DS (control group), 15 sedentary subjects with DS, (SED DS group), nine with DS with low intensity levels of physical activity (LIDS) and 12 with DS with vigorous levels of physical activity (VIDS). The physical activity level was measured using the IPAQ questionnaire. Body composition, hemodynamics and autonomic modulation were also evaluated. Results: The VIDS showed lower BMI than the control and SED DS group. Body fat (\%) was lower in LIDS and VIDS than in the control and SED DS group. The systolic and diastolic arterial pressure values were similar among the groups. When normalized, the frequency domain showed higher LF/HF, higher LF (nu) and lower HF (nu) component of heart rate variability in sedentary DS than in the control group. However, LIDS and VIDS groups showed similar values in LF (nu), HF (nu) and LF/HF than the control group. The symbolic analysis showed greater $0 \mathrm{~V}$ and lower values in $2 \mathrm{LV}$ in sedentary DS group when compared to the control group. However, the LIDS and VIDS did not show difference in $0 \mathrm{~V}$ when compared to the control group. The 2LV was lower in LIDS and VIDS than in the control group, but 2UV was higher in VIDS than in the control group. Conclusion: We conclude that, regardless of the intensity, exercise can promote positive adaptations in the autonomic modulation of DS patients.
\end{abstract}

Keywords: down syndrome; heart rate variability; exercise; intensity.

\section{Introduction}

Down syndrome (trisomy 21) is in one of each $800-1.000$ live-born children, independent of gender, ethnic or racial group, resulting in higher prevalence of other health complications if compared to the general population ${ }^{1}$.

People with Down syndrome (DS) are at high risk for cardiovascular morbidity and mortality and have low levels of cardiovascular fitness ${ }^{2}$. Among the cardiac alterations, chronotropic incompetence and low work capacity are other common findings in people with DS. This was evidenced by the decrease in intrinsic heart rate, evaluated after sympathetic and parasympathetic blockade with atropine and propanolol ${ }^{3}$.

There is compelling evidence that these individuals may exhibit cardiac autonomic dysfunction manifested as a reduced heart rate response to acute sympatho-stimulatory tasks ${ }^{4,5}$. People with DS may show a decrease in peripheral blood flow and a decrease in cardiac output, influenced by baroreflex sensitivity, vagal withdrawal reduced sympathetic activation. Besides the autonomic function, vagal modulation of heart rate is influenced by several physiological factors such as aging, ${ }^{6,7}$. obesity ${ }^{8}$ and aerobic training?.

Low levels of physical fitness is an important health related issue for this population ${ }^{10,11}$. Additionally, overweight and obesity are present in this population. It is known that regular physical activity is an important strategy to ameliorate cardiovascular and respiratory muscle function, prevent obesity and reduce cardiovascular risk factors (i.e., abnormal serum lipid profiles) and decrease the levels of overall mortality and morbidity ${ }^{7}$.
Furthermore, exercise training is considered an effective strategy for improving autonomic function by increasing vagal modulation and baroreflex sensitivity in both healthy and diseased populations. An increase in autonomic modulation is evidenced by an increase in the heart rate variability, both in the frequency and time domains (HF power and SD of all normal R-R intervals, respectively) in healthy people ${ }^{2,12,13}$. In contrast, only limited information on the effects of the practice of exercise training on cardiac autonomic function of persons with DS is available ${ }^{14}$.

The primary purpose of this study was to evaluate the effect of low and vigorous levels of physical activity in anthropometric, hemodynamic and autonomic modulation in persons with DS physically.

\section{Methods}

Sample

We analyzed 13 individuals without DS (control group), 15 sedentary subjects with DS (SED DS group), nine DS subjects with low intensity levels of physical activity (LIDS group) and 12 DS subjects with vigorous levels of physical activity (VIDS). All subjects were males.

To join the study, the subjects were required to show up in the all data collection phases, which consisted of: (1) Public call for the study and explanation of the project; (2) Signature of parents or legal responsible acknowledging their consent 
and agreement; (3) Evaluation of physical activity levels, body composition, hemodynamics and electrocardiogram collection. Those with any disease that could affect blood pressure (heart failure, congenital heart diseases, renovascular diseases, chronic low blood pressure, vasovagal syncope, etc.), or taking any medication for blood pressure were excluded.

All methods used in this study were approved by the institutional ethical board and followed the guidelines in the Declaration of Helsinki (Ethics committee protocol number 65365000).

\section{Evaluation of physical activity levels}

For the analysis of the physical activity levels, the International Physical Activity Questionnaire (IPAQ) was applied. This questionnaire was created by a multinational working group to serve as a tool for epidemiological studies. By analyzing the physical activity performed the previous week, participants were classified as active or sedentary ${ }^{15}$. The energy expenditure in metabolic equivalent tasks (MET) was calculated based on the total minutes of physical activity in the last week (MET-min/ week). The LIDS grouped all subjects with $\leq 600$ MET-min/ week ( $\leq 150 \mathrm{~min} /$ week). The VIDS grouped all subjects with over $600 \mathrm{MET}-\mathrm{min} /$ week (>150 $\mathrm{min} /$ week).

\section{Anthropometric measurements}

Initially, measurements of weight, height and waist circumference were made according to the NHBLI, as previously described ${ }^{16,17}$.

The skinfold measurements were also made to estimate the total body fat $(\%)$. Three skinfolds were performed: Medial Axial, Abdominal and Medial Anterior Quadriceps with a view to analyze the percentage of fat and lean mass ${ }^{18}$.

We used the method of anthropometric evaluation, where all patients admitted were submitted to anamnesis and a complete clinical examination. Data on gender, age, weight, height, body mass index (BMI), blood pressure and waist and hip circumference were recorded ${ }^{19}$. Body mass index (BMI) was calculated by dividing the weight in kilograms $(\mathrm{Kg})$ by the square of the height, measured in meters, $(\mathrm{kg} / \mathrm{m} 2)$. According to the World Health Organization (1998), the following classification was adopted: Overweight: BMI $\geq 25$ and $<29.9 \mathrm{~kg} / \mathrm{m}^{2}$, Obesity grade $1: \mathrm{BMI} \geq 30$ and $<34.9 \mathrm{~kg} / \mathrm{m}^{2}$, Obesity grade $2: \mathrm{BMI}$ $\geq 35$ and $<39.9 \mathrm{~kg} / \mathrm{m}^{2}$, Obesity grade $3: \mathrm{BMI} \geq 40 \mathrm{Kg} / \mathrm{m}^{2}$ (previously termed morbid obesity).

\section{Blood pressure evaluation}

Blood pressure was measured using the auscultatory method, with subjects sitting comfortably for at least 5 minutes on a chair (with their feet flat on the floor), in a calm environment, and with the right arm positioned at the level of the heart. The purpose of analyzing the data in this study was that the mean values obtained in two blood pressure measurements, with 20-minute intervals, could be used. Blood pressure was determined according to the standardization proposed by the VII Brazilian Guidelines for Hypertension $^{20}$. Systolic blood pressure was reported in the first phase of Korotkoff (onset of noise), and diastolic in the $5^{\text {th }}$ phase of Korotkoff (noise disappearance). Values of SBP $\geq 140$ $\mathrm{mmHg}$ and / or DBP $\geq 90 \mathrm{mmHg}$ were used as cut-off points for increased blood pressure ${ }^{21}$.

\section{Heart Rate Variability (HRV)}

The electrocardiogram signal (ECG) acquisition was collected at $\mathrm{kHz}$ sample rate, for a 10-minute period in the supine position, head elevation of $30^{\circ}$. It was done using a protocol with three derivations. To assess the HRV, the temporal series of $\mathrm{RR}$ intervals were registered for posterior analysis (Wincardio Micromed 600hz, Brasilia, DF, Brazil).

Temporal series from the tachogram were related to each selected segment and were quantitatively evaluated considering the values for the HR, total and normalized (nu) powers of low frequency ( $\mathrm{LF}-0.04$ to $0.15 \mathrm{~Hz}$ ) and high frequency ( $\mathrm{HF}-0.15$ to $0.40 \mathrm{~Hz}$ ) components of the HRV. The sympathovagal index (LF/HF) was calculated based on the LF ad HF normalized. Normalized units (nu) were obtained by dividing the power of a given component by the total power (from which VLF was subtracted) and multiplied by $100^{22}$.

In text format, tests were analyzed using the Kubios HRV 2.0 (Biosignal Analysis and Medical Imaging Group, Kuopio, Finland) program in which the ECG signal was processed to obtain the variables related to the HRV in the time and frequency domains. In the time domain, the variables of SDNN (standard deviation of RR intervals) and RMSSD (square root of the mean squared differences between adjacent normal RR intervals, expressed in ms) were chosen. The analysis of the HRV in the frequency domain was performed using Fast Fourier Transform (FFT) in portions of 5 minutes with interpolation of $4 \mathrm{~Hz}$, overlapped by $50 \%$. The bands of interest were low frequency or LF (0:04 to $0: 15 \mathrm{~Hz}$, this component refers predominantly to sympathetic modulation) and high frequency or HF ( 0.15 to $0.4 \mathrm{~Hz}$, referring to parasympathetic modulation $)^{23}$.

Normalized LF and HF components of R-R variability were considered, respectively, as markers of cardiac sympathetic and parasympathetic modulation, and their ratio (LF/HF) considered as an index of the autonomic modulation of the heart. The results were expressed in absolute values ( $\mathrm{HF}$ and $\mathrm{LF} \mathrm{ms}^{2}$ ) and percentage (HFnu and LFnu).

A symbolic analysis was carried out according to the approach previously described and validated by Porta ${ }^{24}$. For this method, the same $5 \mathrm{~min}$ of iRR selected was used. Then, a coarse graining approach based on a uniform quantization procedure was used to transform the iRR series into a sequence of symbols. The length (L) was kept fixed in all analyses. The full range of sequences was uniformly spread over six levels (from 0 to 5), and patterns of length $\mathrm{L}=3$ were constructed. Therefore, each subject and each experimental condition had its range of $i R R$ intervals. The Shannon entropy of the distribution of the patterns was calculated to provide a quantification of the complexity of the pattern distribution. All possible patterns (i.e., 216) were 
grouped without any loss into three families referred to as (1) patterns with no variation. The sequences were spread on six levels, and all possible patterns divided into four groups, consisting of patterns with:

1) no variations $(0 \mathrm{~V}$, three equal symbols, associated with sympathetic modulation); 2 ) one variation ( $1 \mathrm{~V}$, two equal symbols and one different, associated with sympathetic and parasympathetic modulation); 3) two like variations (2LV and associated with parasympathetic modulation ); and 4) two unlike variations (2UV and associated with parasympathetic modulation) .

\section{Statistical analysis}

Regarding data, we used the Shapiro-Wilk Test, which attested a heteroscedastic distribution, and subsequently a One-Way ANOVA with the post-hoc Student-Newman-Keuls test. The significance level was set at $95 \%$ confidence interval or $\mathrm{p}<0.05$ (Graphpad $\AA$ Prism 5). Results showed by mean and standard error.

\section{Results}

Table 1 shows the baseline characteristics of the control group, SED DS, LIDS and VIDS groups. There were no significant differences in age, height, weight, body mass index, and heart rate between the control, SED DS and LIDS groups. The VIDS group showed lower BMI than the control and SED DS groups.
The body fat (\%) was lower in the LIDS and VIDS groups than in the control and SED DS groups. The systolic and diastolic arterial pressures were similar among the groups.

Table 2 shows the comparison of values obtained in the HRV evaluations in the time and frequency domains and symbolic analysis in the investigated subjects. The frequency domain indexes showed that the sedentary DS individuals demonstrated (in the baseline evaluation) similar values in LF and lower values in the absolute HF component than the control group (Table 2). These results, when normalized, showed higher sympathovagal balance, higher normalized LF and lower HF component of the HRV in the sedentary DS group than in the control group. However, the LIDS and VIDS groups showed lower values in the absolute LF component than the sedentary group and, consequently, similar values in normalized values in LF, HF and sympathovagal balance than the control group (Table 2).

The symbolic analysis was expressed in figure 1 and showed greater sympathetic modulation $(0 \mathrm{~V})$ and lower values in parasympathetic modulation ( $2 \mathrm{LV}$ ) in the sedentary DS group when compared to the control group. However, no changes were observed in parasympathetic modulation in $2 \mathrm{UV}$ in sedentary DS when compared to the control group. However, LIDS and VIDS did not show difference in sympathetic modulation (0V) when compared to the control group. The 2LV was lower in the LIDS and VIDS groups than in the control group, but $2 \mathrm{UV}$ was higher in the VIDS group than in the control group. The $1 \mathrm{~V}$ were similar among the groups.

Table 1. Hemodynamics and body composition characteristics of the investigated subjects:

\begin{tabular}{|c|c|c|c|c|}
\hline & $\begin{array}{c}\text { Control } \\
(n=13)\end{array}$ & $\begin{array}{c}\text { Sedentary DS } \\
(n=15)\end{array}$ & $\begin{array}{l}\text { LIDS } \\
(n=9)\end{array}$ & $\begin{array}{l}\text { VIDS } \\
(n=12)\end{array}$ \\
\hline Age (years) & $29 \pm 3.7$ & $26 \pm 6.6$ & $26.1 \pm 1.13$ & $24 \pm 2.2$ \\
\hline Systolic arterial pressure $(\mathrm{mm} / \mathrm{Hg})$ & $119 \pm 6.9$ & $113 \pm 11$ & $113 \pm 2$ & $112 \pm 3$ \\
\hline Diastolic arterial pressure $(\mathrm{mm} / \mathrm{Hg})$ & $80 \pm 2.8$ & $72 \pm 11$ & $73 \pm 3.3$ & $72.8 \pm 2.6$ \\
\hline Weight (kg) & $85 \pm 7$ & $64 \pm 16^{*}$ & $58.8 \pm 3.3^{*}$ & $54.2 \pm 2.6^{* \#}$ \\
\hline Height (cm) & $177 \pm 5.3$ & $150 \pm 6.1^{*}$ & $151.3 \pm 1.5^{*}$ & $149 \pm 2 *$ \\
\hline Body mass index $\left(\mathrm{kg} / \mathrm{m}^{2}\right)$ & $27 \pm 2.6$ & $28 \pm 5.6$ & $25.1 \pm 1.2$ & $23.4 \pm 0.8^{*} \#$ \\
\hline Lean mass $(\%)$ & $77 \pm 4.6$ & $77 \pm 6.1$ & $84.4 \pm 2.4$ & $84.7 \pm 1.3$ \\
\hline Fat mass $(\%)$ & $23 \pm 4.6$ & $23 \pm 6.1$ & $15.6 \pm 2.4$ & $15.3 \pm 1.3$ \\
\hline IPAQ (min/week) & $55 \pm 7$ & $50 \pm 10$ & $165 \pm 30 * \#$ & $315 \pm 45^{*} \#$ \\
\hline MET (MET/min/week) & $165 \pm 24$ & $162 \pm 18$ & $661 \pm 50^{* \#}$ & $1925 \pm 120^{*} \#$ \\
\hline
\end{tabular}

Results showed by mean and standard error; IPAQ, International Physical Activity Questionnaire; MET, metabolic equivalent; One way ANOVA; tukey post-hoc; $* \mathrm{p}<0.05$ vs Control; \# $\mathrm{P}<0.05$ vs. Sedentary DS

Table 2. Comparison of values obtained in the Heart Rate Variability evaluated in the time and frequency domains and Symbolic Analysis in investigated subjects:

\begin{tabular}{lcccc}
\hline & $\begin{array}{c}\text { Control } \\
(\mathbf{n}=\mathbf{1 3})\end{array}$ & $\begin{array}{c}\text { Sedentary DS } \\
(\mathbf{n}=\mathbf{1 5})\end{array}$ & $\begin{array}{c}\text { LIDS } \\
(\mathbf{n}=\mathbf{9})\end{array}$ & $\begin{array}{c}\text { VIDS } \\
(\mathbf{n}=\mathbf{1 2})\end{array}$ \\
\hline Time domain & & & & \\
\hline Total variability $\left(\mathrm{ms}^{2}\right)$ & $2576 \pm 322$ & $1493 \pm 210^{*}$ & $1546 \pm 321$ & $1742 \pm 343$ \\
RR (ms) & $964 \pm 182$ & $805 \pm 43$ & $952 \pm 59$ & $964 \pm 59$ \\
\hline
\end{tabular}




\begin{tabular}{|c|c|c|c|c|}
\hline \multicolumn{5}{|c|}{ Frequency domain } \\
\hline $\mathrm{LF}\left(\mathrm{ms}^{2}\right)$ & $964 \pm 182$ & $906 \pm 258$ & $469 \pm 148^{* \#}$ & $521 \pm 135^{*} \#$ \\
\hline $\mathrm{HF}\left(\mathrm{ms}^{2}\right)$ & $831 \pm 353$ & $489 \pm 332 *$ & $482 \pm 344$ & $644 \pm 233$ \\
\hline LF (nu) & $50 \pm 3.6$ & $70 \pm 2.6^{*}$ & $49 \pm 3.7 \#$ & $45 \pm 1.6$ \\
\hline HF (nu) & $50 \pm 3.6$ & $30 \pm 2.6^{*}$ & $51 \pm 3.7 \#$ & $55 \pm 1.6$ \\
\hline $\mathrm{LF} / \mathrm{HF}$ & $1.2 \pm 0.2$ & $2.87 \pm 0.4^{*}$ & $1.12 \pm 0.21 \#$ & $0.88 \pm 0.05$ \\
\hline \multicolumn{5}{|c|}{ Symbolic analysis } \\
\hline OV $(\%)$ & $19 \pm 3$ & $32 \pm 4.1^{*}$ & $20 \pm 4.9 \#$ & $18 \pm 2.7$ \\
\hline 1V $(\%)$ & $46 \pm 3.6$ & $43 \pm 1.3$ & $45 \pm 1.3$ & $42 \pm 1.7$ \\
\hline $2 \mathrm{LV}(\%)$ & $15 \pm 1.8$ & $7.8 \pm 2.9^{*}$ & $9.12 \pm 2$ & $9.03 \pm 1.3$ \\
\hline 2UV (\%) & $19 \pm 3.0$ & $16 \pm 1.9$ & $23 \pm 2.6$ & $28 \pm 2.5^{*} \#$ \\
\hline
\end{tabular}

$\mathrm{RR}=$ interval RR; ; VARR= PIVLF = very low frequency; $\mathrm{LF}=$ low frequency; $\mathrm{HF}=$ high frequency, $\mathrm{LFNu}=$ normalized low frequency; HFNu = normalized high frequency; $\mathrm{LF} / \mathrm{HF}=$ ratio between $\mathrm{LF}$ and $\mathrm{HF}$ components, $0 \mathrm{~V}=$ three equal symbols , associated with sympathetic modulation); $1 \mathrm{~V}=$ two equal symbols and one different associated with sympathetic and parasympathetic modulation; 2LV two like variations and associated with parasympathetic modulation and $2 \mathrm{UV}$ two unlike variations and associated with parasympathetic modulation.Results showed by mean and standard deviation; One way ANOVA; tukey post-hoc; $*$ p $<0.05$ vs Control; \# $\mathrm{P}<0.05$ vs. Sedentary DS

Figure 1. Symbolic representation of autonomic modulation in the frequency domains among groups
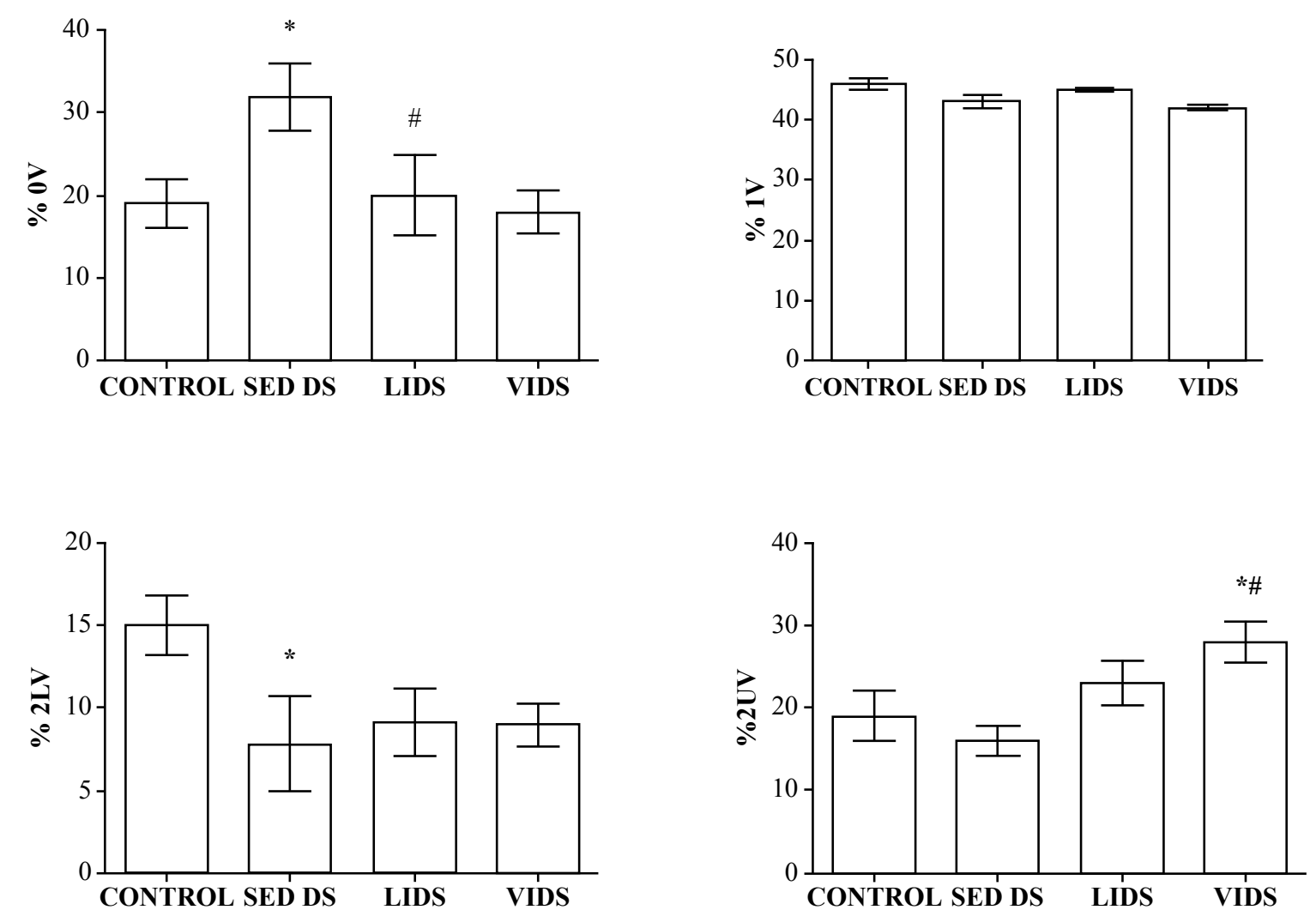

* $\mathrm{p}<0.05$ vs Control; \# $\mathrm{P}<0.05$ vs. Sedentary DS

\section{Discussion}

This study was aimed at evaluating the hemodynamic parameters, autonomic modulation, body composition and physical activity levels in patients with DS. The main finding of the present study was that actives DS groups showed higher values of HRV and lower sympathetic modulation when compared to their sedentary counterparts. A pattern of reduced HRV and reduced sympathetic modulation was also observed in both low-intensity physical activity (LIDS) and vigorous-intensity physical activity (VIDS) DS patients. Additionally, low-intensity and vigorous-intensity DS presented lower BMI and body fat values than the sedentary DS group. These findings strongly suggest that exercise training attenuates autonomic and body composition problems in DS patients regardless of the intensity.

Many studies have shown marked autonomic imbalance in DS patients ${ }^{13,25,26}$. Our study evidenced an autonomic imbalance 
based on the analysis of the HRV in the time and frequency domains, via symbolic analysis. Our data partly corroborates with those presented by Goulopoulou, who had observed a reduced overall variability, suggesting reduced resting vagal modulation in DS patients ${ }^{27}$. However, other studies fail in demonstrate this autonomic modulation imbalance (those studies submitted DS patients to orthostatic stress such as Valsalva maneuver and handgrip strength $)^{5}$. The difference between ${ }^{27}$ these works and our data can be partly explained by the differences of body composition found in our sample (Table 1) and by other secondary pathologies present in the DS patients, such as diabetes and heart diseases.

In DS patients, the prevalence of overweight and obesity has been clearly demonstrated in different research works ${ }^{28}$. This fact can be partially explained due to the sedentary state attested among this population ${ }^{29}$. Both sedentary state and overweight have been correlated with autonomic control imbalance, strongly promoting cardiovascular diseases and pathologies ${ }^{30}$. Previous studies have suggested that the abnormal autonomic function and hemodynamics in obesity are related to hyperinsulinemia ${ }^{29,31}$.

DS inherent abnormalities such as brainstem malfunction ${ }^{32}$, poor growth and encephalic maturation, causing neuronal ${ }^{33,34}$ and dendrite ${ }^{35}$ loss and, other anatomic and genetic disorders can be the cause of the autonomic imbalance. ${ }^{36}$

The clinical relevance of this study lays on the chronotropic incompetence present in the DS patients, leading to increased cardiovascular risk, altogether with the autonomic imbalance, also a risk factor for cardiovascular diseases ${ }^{37}$. The chronotropic incompetence is characterized as an inability to reach $\sim 85 \%$ of the maximal predicted heart rate and delayed heart max achievement ${ }^{38}$. This marked inability to reach the adequate heart rate strongly affects the subject's aerobic capacity, and therefore, normal work capacity, as can be expressed by reduced maximal heart rate and maximal oxygen consumption ${ }^{2,12}$. Also, considering that the life expectancy of DS patients are increasing, the understanding of the risk factors for cardiovascular diseases is critical, once cardiovascular diseases are one of the main causes of death among $\mathrm{DS}^{4,22}$.

Exercise has been widely recognized as a non-pharmacological tool for cardiovascular risk reduction. Beyond that, exercise has also been recognized as a powerful strategy for the improvement of autonomic balance and total heart rate variability, causing reductions in sympathetic tone, increases in vagal tone and better baroreflex sensitivity, as can be seen in many types of populations, such as athletes, diabetics, etc ${ }^{39-}$ ${ }^{41}$. Also, other regulation mechanisms such as the chemo- and metaboreflexes are also involved in the autonomic tone regulation during exercise (REF).

However, its potential benefits are directly connected to the amount of exercise, its intensity, total workload volume and, training frequency ${ }^{31,42}$. Some studies associated high levels of vigorous exercise with improved exercise capacity and disease reduction ${ }^{43,44}$. Our group has been studying the effects of exercise on autonomic modulation in diverse groups, such as chronic kidney disease $e^{45-47}$, elderly ${ }^{48}$ and chronic obstructive pulmonary disease patients (non-published data). Nonetheless, to this date, no studies correlated the level of physical activity with autonomic control of patients with DS.
A powerful tool for indirect but reliable measurement of autonomic modulation is the heart rate variability ${ }^{49}$. This method uses the difference in time between RR intervals to determine autonomic modulation ${ }^{49,50}$. Exercise reduces heart rate by increasing vagal modulation, showed by the heart rate variability through the LF and SDNN indexes ${ }^{49,50}$.

The major limitation of our study lays on the absence of a causal relationship between the physical activity and autonomic modulation in DS patients, mainly because our group did not train those individuals; instead, we just traced their physical activity and correlated with the level of autonomic modulation through HRV measurements.

Regarding autonomic activity, a direct evaluation of the sympathetic activity, such as muscle sympathetic nerve activity, baroreflex activity or catecholamines blood analysis would further enhance the conclusions of this study.

Thus, we conclude that, regardless of the intensity, exercise can promote positive adaptations in the autonomic modulation of DS patients.

\section{References}

1. Myrelid Å, Gustafsson J, Ollars B, Annerén G. Growth charts for Down syndrome from birth to 18 years of age. Arch Dis Child. 2002;87(2):97-103.

2. Fernhall B, Pitetti KH, Rimmer JH, McCubbin JA, Rintala P, Millar AL,et al. Cardiorespiratory capacity of individuals with mental retardation including Down syndrome. Med Sci Sports Exerc. 1996;28(3):366-371.

3. Borresen J, Lambert MI. Autonomic control of heart rate during and after exercise: measurements and implications for monitoring training status. Sports Med. 2008;38(8):633.

4. Figueroa A, Collier SR, Baynard T, Giannopoulou I, Goulopoulou $\mathrm{S}$, Fernhall B. Impaired vagal modulation of heart rate in individuals with Down syndrome. Clin Auton Res. 2005;15(1):45-50.

5. Iellamo F, Galante A, Legramante JM, Lippi ME, Condoluci C, Albertini $\mathrm{G}$, et al. Altered autonomic cardiac regulation in individuals with Down syndrome. Am J Physiol Heart Circ Physiol. 2005;289(6):H2387-H2391.

6. Bittles AH, Bower C, Hussain R, Glasson EJ. The four ages of Down syndrome. Eur J Public Health. 2007;17(2):221-225.

7. Bertapelli F, Pitetti K, Agiovlasitis S, Guerra-Junior G. Overweight and obesity in children and adolescents with Down syndrome - prevalence, determinants, consequences, and interventions: A literature review. Res. Dev. Disabil. 2016;57:181-192.

8. Melville C, Cooper SA, McGrother C, Thorp C, Collacott R. Obesity in adults with Down syndrome: a case-control study. J Intellect Disabil Res. 2005;49(2):125-133.

9. Mostarda C, Wichi R, Sanches IC, Rodrigues B, Angelis K, Irigoyen MC. Hipertensão e modulação autonômica no idoso: papel do exercício físico. Rev Bras Hipertens. 2009;16(1):55-60.

10. Lanfranchi S, Jerman O, Dal Pont E, Alberti A, Vianello R. Executive function in adolescents with Down syndrome. J Intellect Disabil Res. 2010;54(4):308-319. 
11. Guedes DP, Guedes JERP, Barbosa DS, Oliveira JAd. Levels of regular physical activity in adolescents. Rev Bras Med Esporte. 2001;7(6):187-199.

12. Fernhall B, McCubbin JA, Pitetti KH, Rintala P, Rimmer $\mathrm{JH}$, Millar AL, et al. Prediction of maximal heart rate in individuals with mental retardation. Med Sci Sports Exerc. 2001;33(10):1655-1660.

13. Baynard T, Pitetti KH, Guerra M, Fernhall B. Heart rate variability at rest and during exercise in persons with Down syndrome. Arch Phys Med Rehabil. 2004;85(8):1285-1290.

14. Agiovlasitis S, Collier SR, Baynard T, Echols GH, Goulopoulou $\mathrm{S}$, Figuero A, et al. Autonomic response to upright tilt in people with and without Down syndrome. Research in developmental disabilities. 2010;31(3):857-863.

15. Matsudo S, Araújo T, Matsudo V, Andrade D, Andrade E, Oliveira LC, et al. Questionário Internacional De Atividade Física (Ipaq): Estupo De Validade E Reprodutibilidade No Brasil. Rev. bras. ativ. fís. saúde. 2012;6(2):5-18.

16. Vainio H, Bianchini F. Weight control and physical activity. Iarc; 2002.

17. Klein S, Allison DB, Heymsfield SB, Kelley DE, Leibel RL, Nonas $\mathrm{C}$, et al. Waist circumference and cardiometabolic risk: a consensus statement from shaping America's health: Association for Weight Management and Obesity Prevention; NAASO, the Obesity Society; the American Society for Nutrition; and the American Diabetes Association. Obesity. 2007;15(5):1061-1067.

18. Kang J, Chaloupka EC, Mastrangelo MA, Biren GB, Robertson RJ. Physiological comparisons among three maximal treadmill exercise protocols in trained and untrained individuals. Eur J Appl Physiol. 2001;84(4):291-295.

19. Rezende FAC, Rosado LEFPL, Ribeiro RCL, Vidigal FC, Vasques ACJ, Bonard IS, et al. Índice de massa corporal e circunferência abdominal: associação com fatores de risco cardiovascular. Arq Bras Cardiol. 2006;87(6):728-734.

20. SBC SBdC. 7a Diretriz Brasileira de Hipertensão Arterial. Arq Bras Cardiol. 2016;107(3):1-82.

21. Maia VBdS, Veras AB, de Souza Filho MD. Pressão arterial, excesso de peso e nível de atividade física em estudantes de universidade pública. Arq Bras Cardiol. 2010;95(2):192-199.

22. Mostarda C, Rodrigues B, Vane M, Moreira ED, Rosa KT, Moraes-Silva IC, et al. Autonomic impairment after myocardial infarction: role in cardiac remodelling and mortality. Clin Exp Pharmacol Physiol. 2010;37(4):447-452.

23. Miranda JMdQ, Dias LdC, Mostarda CT, Angelis KD, Figueira Junior AJ, Wichi RB. Effect of strength training on cardiovascular variables in overweight adolescents. Rev Bras Med Esporte. 2014;20(2):125-130.

24. Porta A, Tobaldini E, Guzzetti S, Furlan R, Montano N, GnecchiRuscone T. Assessment of cardiac autonomic modulation during graded head-up tilt by symbolic analysis of heart rate variability. Am J Physiol Heart Circ Physiol . 2007;293(1):H702-H708.

25. Ferri R, Curzi-Dascalova L, Del Gracco S, Elia M, Musumeci S, Pettinato S. Heart rate variability and apnea during sleep in Down syndrome. J Sleep Res. 1998;7(4):282-287.

26. Junior DEB, Lopes LLM, Accioly MF, Riberiro MF, Ferreira AA, Teixeira VPA, et al. Autonomic modulation of assessment in individuals with Down syndrome in hippotherapy. ConScientiae Saúde. 2016;15(3):433.

27. Goulopoulou S, Baynard T, Collier S, Giannopoulou I, Figueroa A, Beets M, et al. Cardiac autonomic control in individuals with Down syndrome. Am J Ment Retard. 2006;111(1):27-34.

28. Silva NM, Gomes Filho A, da SILVA SF, Fernandes Filho J. Indicadores antropométricos de obesidade em portadores da síndrome de Down entre 15 e 44 anos. Rev bras Educ Fís Esporte. 2009;23(4):415-424.

29. Seron BB, Silva RAC, Greguol M. Effects of two programs of exercise on body composition of adolescents with Down syndrome. Rev Paul Pediatr. 2014;32(1):92-98.

30. Lopes HF, Egan BM. Desequilíbrio autonômico e síndrome metabólica: parceiros patológicos em uma pandemia global emergente. Arq Bras Cardiol. 2006;87(4):538-547.

31. Florentino Neto J, Pontes LMd, Fernandes Filho J. Alterações na composição corporal decorrentes de um treinamento de musculação em portadores de síndrome de down. Rev Bras Med Esporte. 2010;16(1):09-12.

32. Kazan HM, Samelli AG, Neves-Lobo IF, Magliaro FCL, Limongi SCO, Matas CG. Electrophysiological characterization of hearing in individuals with Down syndrome. Paper presented at: CoDAS2016.

33. Giagkoudaki F, Dimitros E, Kouidi E, Deligiannis A. Effects of exercise training on heart-rate-variability indices in individuals with Down syndrome. J Sport Rehabil. 2010;19(2):173-183.

34. Al-Biltagi M, Serag AR, Hefidah MM, Mabrouk MM. Evaluation of cardiac functions with Doppler echocardiography in children with Down syndrome and anatomically normal heart. Cardiol Young. 2013;23(2):174-180.

35. Benavides-Piccione R, Ballesteros-Yanez I, de Lagrán MM, Elston G, Estivill X, Fillat C, et al. On dendrites in Down syndrome and DS murine models: a spiny way to learn. Prog. Neurobiol. 2004;74(2):111-126.

36. Mendonca GV, Pereira FD, Fernhall B. Reduced exercise capacity in persons with Down syndrome: cause, effect, and management. Ther Clin Risk Manag. 2010;6:601.

37. Guerra M, Llorens N, Fernhall B. Chronotropic incompetence in persons with down syndrome. Arch Phys Med Rehabil. 2003;84(11):1604-1608.

38. Gentlesk PJ, Markwood TT, Atwood JE. Chronotropic incompetence in a young adult: case report and literature review. CHEST Journal. 2004;125(1):297-301.

39. Ordonez FJ, Rosety M, Rosety-Rodriguez M. Influence of 12week exercise training on fat mass percentage in adolescents with Down syndrome. Med Sci Monit. 2006;12(10):CR416-CR419.

40. Heller T, Hsieh K, Rimmer J. Barriers and supports for exercise participation among adults with Down syndrome. J Gerontol Soc Work. 2003;38(1-2):161-178.

41. Roche F, Pichot V, Da Costa A, Isaaz K, Costes F, Dall'Acqua T, et al. Chronotropic incompetence response to exercise in congestive heart failure, relationship with the cardiac autonomic status. Clin Physiol Funct Imaging. 2001;21(3):335-342.

42. Guedes DP, Guedes J. Atividade física, aptidão cardiorrespiratória, composição da dieta e fatores de risco predisponentes às doenças cardiovasculares. Arq Bras Cardiol. 2001;77(3):243-250. 
43. Pollock ML, Gettman LR, Milesis CA, Bah MD, Durstine L, Johnson RB. Effects of frequency and duration of training on attrition and incidence of injury. Med Sci Sports. 1976;9(1):31-36.

44. Pollock ML, Franklin BA, Balady GJ, Chaitman BL, Fleg JL, Fletcher B, et al. Resistance exercise in individuals with and without cardiovascular disease. Circulation. 2000;101(7):828-833.

45. Barroso R, Silva-Filho AC, Dias CJ, Soares NJr, Mostarda A, Azoulbel LA, et al. Effect of exercise training in heart rate variability, anxiety, depression, and sleep quality in kidney recipients: A preliminary study. J Health Psychol. 2016:1359105316676329.

46. Dias CJM, Azoubel LA, Costa HA, Maia EC, Rodrigues B, Silva-Filho AC, et al. Autonomic modulation analysis in active and sedentary kidney transplanted recipients. Clin Exp Pharmacol Physiol. 2015;42(12):1239-1244.

47. Dias CJ, Anaisse-Azoubel LM, Carneiro ECRL, Rodrigues B, Silva-Filho AC, Irigoyen MC, et al. Comparison of Baroreflex Response to Acute Sessions of Strength and Aerobic Exercises in Kidney Recipients. J Exerc Physiol Online. 2017;20(5).

48. de Carvalho CA, Soares L, Sevílio Jr M, Silva-Filho AC, Dias $\mathrm{CJ}$, Rodrigues B, et al. Effect of exercise in air-conditioned and non-air-conditioned environment in cardiac autonomic control. J Sports Med Phys Fitness. 2017;57(7-8):1080.

49. Malik M. Clinical guide to cardiac autonomic tests. Vol 206: Springer Science \& Business Media; 1998.
50. Malik M. Heart rate variability. Annals of Noninvasive Electrocardiology. 1996;1(2):151-181.

\section{Acknowledgements}

We would like to thank those who joined this research project and their families. Cristiano Mostarda received grants from CNPq (Universal 442374/2014-3) and FAPEMA (Universal 00358/15).

\section{*Corresponding author}

Cristiano Teixeira Mostarda, Av. dos Portugueses, 1966, Cidade Universitária Dom Delgado, Dept. de Educação Física.

Email: cristiano.mostarda@gmail.com

Manuscript received on September 14, 2017

Manuscript accepted on December 17, 2017

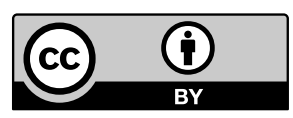

Motriz. The Journal of Physical Education. UNESP. Rio Claro, SP, Brazil - eISSN: 1980-6574 - under a license Creative Commons - Version 4.0 


\section{ERRATUM}

In the article "Impacts of low or vigorous levels of physical activity on body composition, hemodynamics and autonomic modulation in Down syndrome subjects", published in volume 24, number 1, 2018: DOI: 10.1590/S1980-6574201800010013 and identification: e1018132.

\section{Where it was written}

${ }^{1}$ Universidade Federal do Maranhão, UFMA, Laboratório de Adaptação Cardiovascular ao Exercício Cardiovascular, LACORE, São Luís, MA, Brazil; ' 2Universidade Federal do Maranhão, UFMA, São Luís, MA, Brazil;

${ }^{3}$ Universidade Estadual de Campinas, UNICAMP, Faculdade de Educação Física, Campinas, SP, Brazil

\section{Should read:}

${ }^{1}$ Universidade Federal do Maranhão, UFMA, Laboratory of Cardiovascular Adaptations to Exercise, LACORE, São Luís, MA, Brazil; ${ }^{2}$ Universidade Federal do Maranhão, Curso de Educação Física, Campus Pinheiro, MA, Brazil, ${ }^{3}$ Universidade Estadual de Campinas, UNICAMP, Faculty of Physical Education, Campinas, SP, Brazil 